\title{
Analysis of Structural Strength and Stacking Compressive Properties for LLDPE Packaging Box
}

\author{
Lixin $\mathrm{Qi}^{1, \mathrm{a}}$, Jinming Zhang ${ }^{1, \mathrm{~b}}$, Ke Chen $^{1, \mathrm{c}}$, Shutao Huang ${ }^{1, \mathrm{~d}}$ \\ ${ }^{1}$ School of Automobile and Transportation, Shenyang Ligong University, Shenyang, 110159, China \\ a syqlxyy@126.com, b1053334576@qq.com, chen_ke@163.com, ${ }^{\mathrm{a}}$ syithst@163.com
}

Keywords: LLDPE; packaging box; structural strength; stacking; compressive resistance

\begin{abstract}
Structural strength and stacking compressive performance of packaging box is an important performance index. Finite element analysis on structural strength and stacking compressive performance was completed by ANSYS Workbench for a new type of LLDPE packaging box, and the strength of packaging box was evaluated based on the deformation nephogram, the stress nephogram and the safety coefficient. The analysis results show that the structural strength of the packaging box can meet the performance requirements. The analysis method and calculation results have practical reference value to design and strength analysis of the packaging boxes.
\end{abstract}

\section{Introduction}

The packaging box is the important equipment for storage and transportation of materials, to meet the requirements of light weight, high strength, easy handling, stacking compressive and other aspects. However, for the high reliable packaging box with complex shape, the simple use of formulas to calculate its strength cannot achieve the desired accuracy[1,2].

Linear low density polyethylene (LLDPE for short) as a new material to make packaging boxes has the incomparable advantage of other materials in lightweight and sealing performance[3,4]. In this paper, structural safety and stacking compressive strength of LLDPE packaging box were analyzed under different loading, and to evaluate its structural strength by studying the deformation nephogram, stress nephogram and safety coefficient.

\section{The Structural Design of the Packaging Box}

The shape and structural of the packaging box is shown in Fig 1, its overall dimension is $400 \mathrm{~mm} \times 300 \mathrm{~mm} \times 400 \mathrm{~mm}$ with thickness is $5 \mathrm{~mm}$. The weight of the packaging box is $5 \mathrm{~kg}$ and it can carry $20 \mathrm{~kg}$. Design requirements the maximum stacking tier is 7 .

\section{Theoretical Foundation of Strength Analysis}

Using static structural module of ANSYS Workbench software, the effect of the changeless inertial load and the approximate equivalent static load on structures can be calculated[5], so the loading status of the packaging box was simulated by the software.

For a linear static structural analysis, the deformation $\{\boldsymbol{X}\}$ can be solved by the matrix equation:

$[\boldsymbol{K}]\{\boldsymbol{X}\}=\{\boldsymbol{F}\}$

In which, $[K]$ is the stiffness matrix; $\{F\}$ is the force of static loading on the model. 


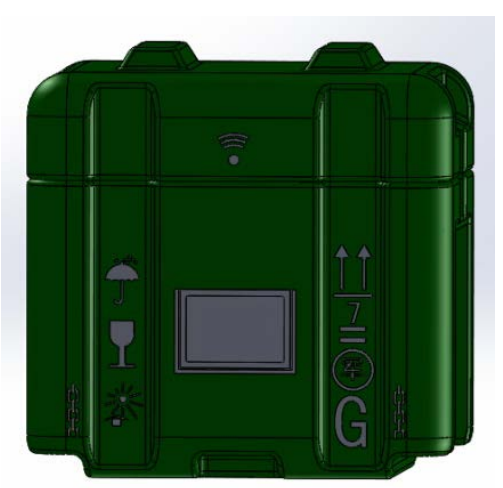

Fig 1 3D model of the packaging box

\section{The Compressive Properties Analysis of the Packaging Box}

The compression properties analysis is applicable to the assessment of the compressive resistance of the packaging box and the ability to protect the equipment parts inside the packaging box. According to the test requirement, a horizontal pressure plate which is bigger than the box cover is placed on the top of the box cover, pressure on the box cover gradually, until the packaging box is broken. Considering the packaging box positioning in transportation and stacking storage, a locating lug boss is arranged at the top of the box cover combined with locating slot which is at the bottom of the box body to achieve the self-positioning. In the packaging box compressive properties analysis, the pressure plate is applied on the locating lug boss directly, the actual loading area of the box cover is much smaller than ideal loading area, which will reduce the compression resistance of the box cover, so the maximum number of stacking is 3 in the resistance capability test.

Create Finite Element Model. The packaging box is composed of linear low density polyethylene made by rotational moulding, the material model is linear elastic model. The material properties are shown in Table 1.

Table 1 the properties of LLDPE

\begin{tabular}{c|c|c|c|c|c}
\hline $\begin{array}{c}\text { Elastic } \\
\text { Modulus }\end{array}$ & $\begin{array}{c}\text { Poisson's } \\
\text { Ratio }\end{array}$ & Density & $\begin{array}{c}\text { Bulk } \\
\text { Modulus }\end{array}$ & $\begin{array}{c}\text { Shear } \\
\text { Modulus }\end{array}$ & $\begin{array}{c}\text { Tensile Yield } \\
\text { Strength }\end{array}$ \\
\hline $600 \mathrm{MPa}$ & 0.4 & $932 \mathrm{~kg} / \mathrm{m}^{3}$ & $1000 \mathrm{MPa}$ & $214.29 \mathrm{MPa}$ & $16.8 \mathrm{MPa}$ \\
\hline
\end{tabular}

To simplify the FE model, remove the handles, hinges, etc, which have no effect on the strength of the packaging box, and add the weight of these parts to loading the box. Using Solid186 solid element, the element supports plasticity, hyper elasticity, creep, stress stiffening, large deflection, and large strain capabilities. The element size is $8 \mathrm{~mm}$; the mesh is divided using adaptive meshing method. 313202 nodes and 166527 elements are generated. The finite element model of the packaging box compression properties analysis is shown in Fig.2. Bond contact was set between the box cover, body and the pressure plate. Adding pressure force which equivalent to the weight of two full crates on the pressure plate. The fixed constraint is set at the bottom of the packaging box, and simulation is carried out using ANSYS Workbench.

Analysis of Compressive Properties. The deformation nephogram that obtained by simulation is shown in Fig.3 and the stress nephogram is shown in Fig.4. From Fig.3, the maximum deformation of the box is $4.74 \mathrm{~mm}$, near the center of the box cover. The position is far away from the support, so this is consistent with the actual situation. Fig.4 shows that the maximum stress of the box is14.5MPa, near the root of the positioning boss, based on actual experience, this location prone to stress concentration, therefore, the analysis result accords with the actual. Since the maximum stress is less than the tensile yield strength of LLDPE, the structural strength to meet the loading requirements.

From the analysis, the minimum safety coefficient of the packaging box is 1.16. Even though it meets the strength requirements, but the safety margin is small. This is due to the unreasonable of loading area. Therefore, it's important to pay attention to the loading distribution when the different kinds of packaging boxes are put on the other box cover, and try to avoid stress concentration. 


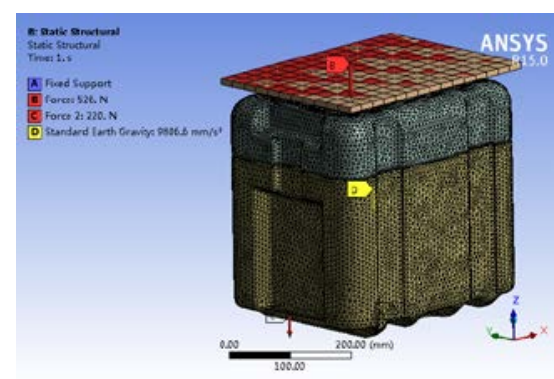

Fig.2 The FE model of compressive properties analysis

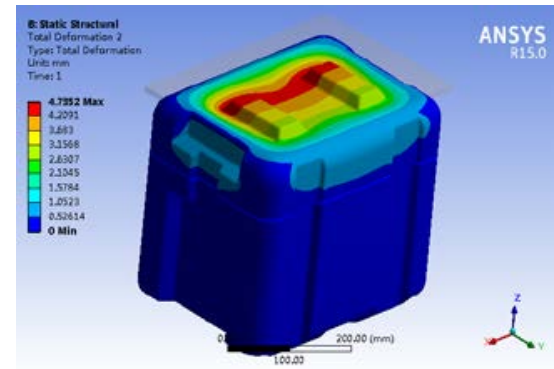

Fig.3 The compressive deformation nephogram

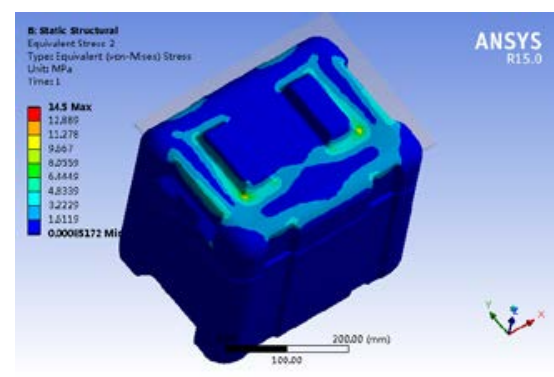

Fig.4 The compressive stress nephogram

\section{The Packaging box Stacking Compressive Strength Analysis}

The compression properties analysis is applicable to the assessment of the load capacity the bottom of the packing box when the packing box transportation and stacking. The full load packaging box is placed in the measured box one by one, and make sure the loading area of the measured box cover is same with the real working condition. The number of stacking tier is 7 .

Create Finite Element Model. Due to the requirement of the actual loading area is the same as the design loading area of the measured box cover, so in order to ensure the authenticity of the simulation a full loading box is put on the measured box cover. The weight of 5 full load boxes and load box cover are equivalent to a uniform loading which acting on the joint face between the box cover and box body for reduce computational effort. Adding uniformly distributed load that simulate the effect of things inside the box to the measured box in the loading box and the measured box bottom. Bond contact and standard Earth Gravity is added to the model, and fixed the bottom of the measured box. 499230 nodes and 266186 elements were generated. The FE model of the stacking compressive strength analysis is shown in Fig.5.

Analysis of Stacking Compressive Properties. The deformation nephogram and the stress nephogram of the measured box are shown in Fig.6 and Fig.7.

From Fig.6, the maximum deformation of the box is $2.16 \mathrm{~mm}$. Because of the asymmetric design of the positioning bosses on the box cover in the width direction, therefore, the point near the root of the positioning bosses, far from the support, has the largest amount of deformation.

As shown in Fig. 7, the maximum equivalent stress of the box is $9.37 \mathrm{MPa}$, is located in the vertices of the measured box cover positioning slot. The place is easy to produce stress concentration. The result shows that the maximum equivalent stress is less than the tensile yield strength of LLDPE, and the safety coefficient is 1.79 under the limit working conditions, therefore the structural strength not only to meet the stacking requirements, but also withstand short-term overload.

The Fig.8 and Fig.9 show the relationship curve between stacking tier and maximum deformation or equivalent stress of the package box. The figure clearly informed the effect on the bottom package box with the stacking layers increasing.

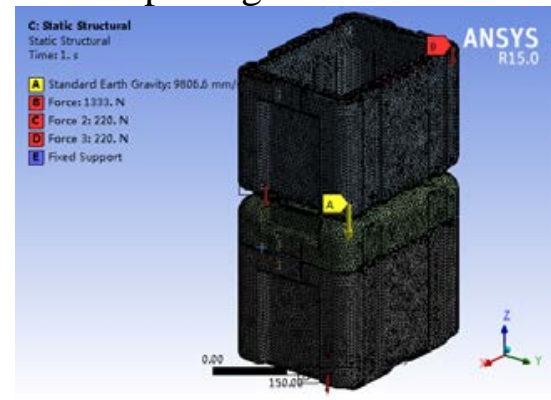

Fig.5 The FE model of stacking compressive strength analysis

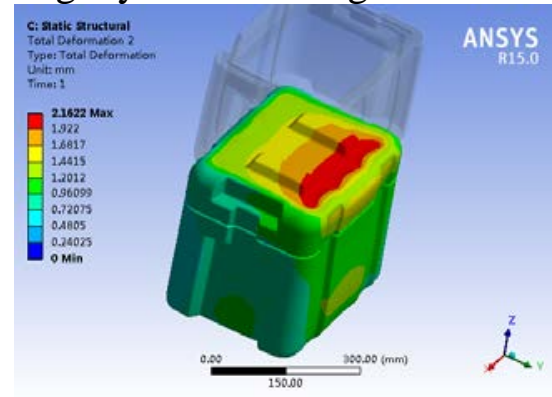

Fig.6 The stacking deformation nephogram

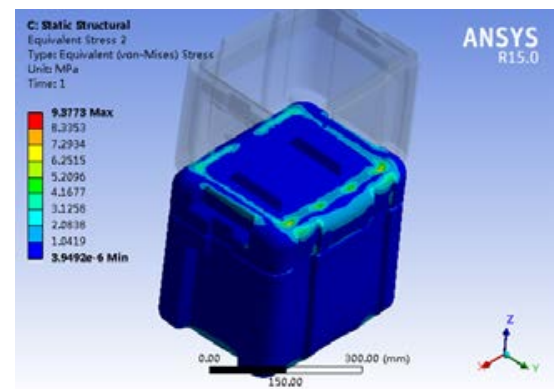

Fig.7 The stacking stress nephogram 


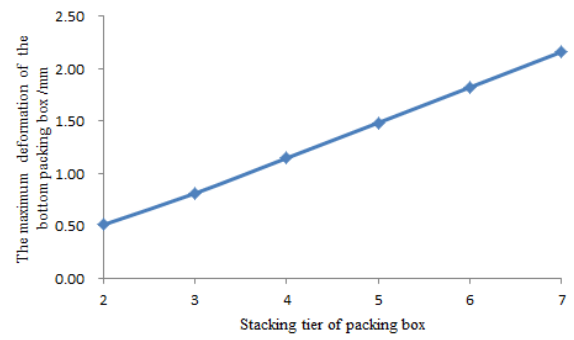

Fig.8 The curve of stacking tier vs maximum deformation

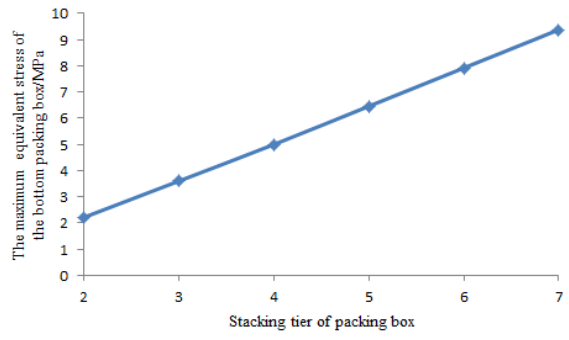

Fig.9 The curve of stacking tier vs maximum equivalent stress

\section{Conclusion}

(1) In this paper, the analysis of structural strength and stacking compressive strength for a new type of LLDPE packaging box was completed by ANSYS Workbench static structural module, and the deformation nephogram, stress nephogram and the safety coefficient were obtained under different load. The analysis results showed that the structural of the packaging box meet the performance requirements.

(2) Under stacking condition of the same type of packaging boxes, the safety margin of the packaging box is more abundant, so it can bearing short-term overload. However, under stacking condition of the different kinds of packaging boxes, there is concentration of stress at the positioning bosses of the box cover, which will greatly reduce the carrying capacity of the box cover. In order to make full use of box body strength, adding supporting pad to eliminate the offset of the actual bearing parts.

(3) Because there are some of the same structures for the LLDPE packaging boxes, this article has practical reference value to design and strength analysis of the packaging boxes.

\section{Reference}

[1] Yonggen Yao, Manru Chen: Packaging Engineering, Vol. 31 (2010) No.23, p. 60-63.(in Chinese)

[2] Jinchen Tian, Yi Qian: Packaging and Food Machinery, (2014) No.4, p.1-4. (in Chinese)

[3] Xiao Dekai, Shengping Wang and Hongbo Xu: Engineering Plastics Application, Vol. 33 (2005) No.5, p.9-12. (in Chinese)

[4] Xianfeng Yu, Hongbing Zhang: Lu Qi Petrochemical Technology, Vol. 32 (2004) No.3, p.204-207. (in Chinese)

[5] Guilong Ling, Jinbin Ding and Zheng Wen: ANSYS Workbench 13.0 from Entry to the Master (Tsinghua University Press, China, 2012). (in Chinese) 